\title{
Factores asociados a síndrome confusional agudo en adultos mayores internados en emergencia de un hospital terciario
} Acute confusional state-associated factors in older adults admitted to a tertiary hospital emergency unit

\author{
José Percy Amado Tineo', Luis Alberto Chucas Ascencio', \\ César Rigoberto Rojas Moya', Silvia Pintado Caballero', \\ Carlos Alberto Cerrón Aguilar ${ }^{1}$, Rolando Vásquez Alva ${ }^{1}$ \\ 'Servicio de Emergencia Adultos, Hospital Nacional Edgardo Rebagliati Martins, EsSalud, Lima, Perú.
}

\begin{abstract}
Resumen
Introducción: El sindrome confusional agudo es un problema frecuente en el adulto mayor, incrementado por patologías agudas. Objetivos: Determinar la frecuencia del sindrome confusional agudo en adultos mayores no críticos hospitalizados en un servicio de emergencia e identificar factores asociados. Diseño: Estudio transversal. Lugar: Servicio de Emergencia del Hospital Nacional Edgardo Rebagliati Martins, Lima, Perú. Participantes: Pacientes de 60 años o más. Intervenciones: Siguiendo normas de buenas prácticas clínicas, se entrevistó entre mayo y agosto 2010 a paciente/cuidador de 172 pacientes de 60 años o más, hospitalizados en salas de emergencia; se revisó la historia clínica. Principales medidas de resultados: Detectar sindrome confusional agudo mediante el Confussion Assessment Method. Resultados: La frecuencia de sindrome confusional agudo fue $34,9 \%$, según categoría de adulto mayor: enfermo $6 \%$, frágil $28 \%$, complejo $58 \%$ ( $p<0,001$ ). Se encontró antecedente de demencia en $30 \%$ de pacientes con síndrome confusional, deterioro cognitivo previo en $45 \%$, sindrome confusional previo en $40,2 \%$, dependencia funcional parcial $46 \%$ y total $39 \%$, deterioro cognitivo moderado $27 \%$ y severo $40 \%$. El $41,7 \%$ de pacientes con síndrome confusional presentó deshidratación moderadasevera ( $p=0,001)$ y $20 \%$ sepsis, como diagnósticos de ingreso $(p=0,003)$. Las patologias de ingreso más frecuentes fueron infecciosas, cardiovasculares y metabólicas. No se encontró diferencia significativa en sexo, grado de instrucción, estado civil, pluripatología, hospitalización previa, polifarmacia, estado nutricional, presencia de sonda urinaria o nasogástrica, destino al egreso de emergencia ni estancia hospitalaria. Conclusiones: Existe alta frecuencia de síndrome confusional agudo en adultos mayores hospitalizados en servicios de emergencia, siendo factores asociados la demencia, deterioro cognitivo previo, confusión previa, dependencia funcional, categoria geriátrico complejo, deshidratación moderada-severa y sepsis.
\end{abstract}

Palabras clave: Adulto mayor hospitalizado, síndrome confusional agudo.

\section{Abstract}

Introduction: Acute confusional state is a frequent problem in the elderly and is increased by acute pathologies. Objectives: To determine the frequency of acute confusional state in non-critical elderly emergency service inpatients and to identify associated factors. Design: Cross sectional study. Setting: Emergency Unit, Hospital Nacional Edgardo Rebagliati Martins, Lima, Peru. Participants: Sixty-year-old or older patients hospitalized in an emergency unit. Interventions: Between May and August 2010 and according to procedures of good clinical practices, 172 60-year-old or older patients hospitalized in an emergency unit or their caregivers were interviewed and clinical records were reviewed. Main outcome measures: Acute confusional state detection with the Confusion Assessment Method. Results: The frequency of acute confusional state according to category of elderly was $34.9 \%$ : ill $6 \%$, fragile $28 \%$ and complex $58 \%(p<0,001)$. There was history of dementia in $30 \%$ of patients with acute confusional state, cognitive previous deterioration in $45 \%$, previous acute confusional state in $40.2 \%$, functional partial dependence in $46 \%$ and total dependence in $39 \%$, cognitive moderate deterioration in $27 \%$ and severe in $40 \% ; 41.7 \%$ of patients with acute confusional state presented moderate - severe dehydration $(p=0.001)$ and $20 \%$ sepsis at admission $(p=0.003)$. The most frequent pathologies at admission were infectious, cardiovascular and metabolic. There was no significant difference in sex, instruction degree, marital status, pluripathology, previous hospitalization, polymedication, nutritional condition, use of urinary or nasogastric tubes, destiny at discharge, or hospital stay. Conclusions: High frequency of acute confusional state existed in elderly patients, being associated factors dementia, previous cognitive deterioration, previous confusional state, functional dependence, complex geriatric category, moderate - severe dehydration and sepsis.

Keywords: Older inpatient, acute confusional state.

An Fac med. 2013;74(3):193-7 


\section{INTRODUCCIÓN}

El avance científico ha permitido el incremento de la expectativa de vida de las personas, habiéndose producido un aumento significativo del número de personas adultas mayores en el mundo. Para el 2007, en Perú los mayores de 60 años constituyeron $6,4 \%$ de la población nacional (casi $80 \%$ más respecto a 1970), concentrándose en Lima y Callao el $32 \%$ de este grupo ${ }^{(1,2)}$.

El adulto mayor tiene características especiales, siendo más vulnerable debido a la elevada tasa de morbilidad crónica y discapacidad, requiriendo especial atención sociosanitaria ${ }^{(3,4)}$.

Entre los problemas mentales más frecuentes en el adulto mayor se encuentra el síndrome confusional agudo (SCA), con frecuencias de 14 a $56 \%$ en pacientes hospitalizados -muchas veces infradiagnosticado-, asociado a mayor morbimortalidad, estancia hospitalaria, institucionalización y deterioro funcional ${ }^{(5,6)}$. Estudios previos han determinado como factores de riesgo para desarrollar SCA a la demencia, polifarmacia o uso de psicofármacos ${ }^{(7-12)}$.

Se define SCA como el conjunto de alteraciones cognitivas de inicio brusco y curso fluctuante, caracterizado por compromiso de atención, nivel de conciencia, claridad de pensamiento y orientación; se le debe considerar una urgencia médica, cuyo diagnóstico y tratamiento precoz previenen complicaciones $^{(1,4)}$.

El SCA en la mayoría de casos es multifactorial y su enfoque diagnóstico incluye anamnesis adecuada, exploración física exhaustiva y estudios complementarios que permitan determinar causas predisponentes y desencadenantes. Sin embargo, existen métodos e instrumentos estandarizados y validados para la detección clínica de este síndrome ${ }^{(1,4,7)}$.

En nuestro medio, las patologías agudas sobreagregadas, junto al inadecuado sistema de salud, desbordan la capacidad de atención en los establecimientos de referencia, siendo los servi- cios de urgencias y hospitalización los más afectados ${ }^{(3,4)}$.

En el presente artículo se plantea como objetivos determinar la frecuencia y los factores asociados al síndrome confusional agudo en adultos mayores no críticos internados en la emergencia del Hospital Nacional Edgardo Rebagliati Martins, 2010.

La importancia del presente estudio radica en determinar la frecuencia de talario e identificar factores asociados para su prevención y control, así como iniciar nuevas investigaciones en este campo.

\section{MÉTODOS}

Este estudio transversal fue realizado en pacientes de 60 años o más, que permanecieron por más de 24 horas en salas de observación de la emergencia del Hospital Nacional Edgardo Rebagliati Martins-EsSalud, Jesús María, Lima, Perú.

Se evaluó consecutivamente 189 pacientes admitidos por problemas médicos, de mayo a agosto del 2010, excluyendo a 17 pacientes por haber egresado en las 24 horas previas de algún servicio hospitalario, presentar condición crítica de salud, asociación con abstinencia alcohólica, haberse retirado por alta voluntaria o preseneste problema en nuestro medio hospi-

tar datos incompletos. Quedaron como muestra válida 172 pacientes.

Se realizó entrevista al paciente o al cuidador, evaluación geriátrica integral y revisión de la historia clínica hospitalaria. Se siguió las normas de buenas prácticas clínicas y tuvo la aprobación de la oficina de investigación institucional.

Se consideró antecedente de demencia, deterioro cognitivo y síndrome confusional previo al comunicado por el cuidador u obtenido en la historia clínica. El estado nutricional fue clasificado según la circunferencia del brazo no dominante, medido en la mitad, hacia el 0,1 cm más cercano. Para evaluar el estado funcional se empleó la prueba de Katz para actividades básicas de la vida diaria, y para estado el cognitivo la prueba de Pfeiffer.

Para diagnosticar síndrome confusional agudo se usó como instrumento el Confusion Assessment Method (CAM), aplicado por uno de cinco médicos internistas capacitados, durante las primeras 24 horas de admisión del paciente al área de estudio.

El CAM es una encuesta de cuatro ítems, que se realiza en menos de cinco minutos y ha sido validada comparándola con evaluación psiquiátrica como referencia estándar, con alta sensibilidad (de 94 a 100\%) y especificidad (90 a $95 \%)(4,13)$.

Tabla 1. Factores asociados a sindrome confusional agudo.

\begin{tabular}{|c|c|c|c|c|c|}
\hline \multirow[t]{2}{*}{ Característica } & \multicolumn{2}{|c|}{$\begin{array}{c}\text { Confusión } \\
\text { (60 pacientes) }\end{array}$} & \multicolumn{2}{|c|}{$\begin{array}{c}\text { No confusión } \\
\text { (112 pacientes) }\end{array}$} & \multirow[t]{2}{*}{ Valor $p$} \\
\hline & $\mathrm{N}$ & $\%$ & $\mathrm{~N}$ & $\%$ & \\
\hline \multicolumn{6}{|c|}{ Antecedente de demencia } \\
\hline Sí & 18 & 30 & 7 & 6,3 & \multirow[t]{2}{*}{0,000} \\
\hline No & 42 & 70 & 105 & 93,7 & \\
\hline \multicolumn{6}{|c|}{ Deterioro cognitivo previo } \\
\hline Sí & 27 & 45 & 16 & 14,3 & \multirow[t]{2}{*}{0,000} \\
\hline No & 33 & 55 & 96 & 75,7 & \\
\hline \multicolumn{6}{|l|}{ Confusión previa } \\
\hline Sí & 41 & 40,2 & 25 & 18,4 & \multirow[t]{2}{*}{0,000} \\
\hline No & 61 & 59,8 & 111 & 81,6 & \\
\hline
\end{tabular}


Se consideró condiciones básicas para el diagnóstico de SCA el inicio agudo con curso fluctuante y la inatención, además de uno de los siguientes criterios: pensamiento desorganizado o alteración del nivel de conciencia ${ }^{(4,13)}$.

Los datos fueron codificados y procesados con el programa SPSS 17.00, empleando medidas de distribución de frecuencia, tendencia central, chi cuadrado y prueba t de student.

\section{RESULTADOS}

De los 172 pacientes, $56 \%$ fue de sexo femenino, edad promedio 78,5 años, desempleado $93 \%$ y $94 \%$, procedía de los distritos de Lima Metropolitana, con promedio de estancia en emergencia 2,8 días.

La frecuencia de síndrome confusional agudo fue $34,9 \%$. Los factores y condiciones clínicas asociadas a síndrome confusional agudo son descritos en las tablas 1 y 2. La frecuencia de síndrome confusional agudo según categoría clínica del adulto mayor fue: enfermo $6 \%$, frágil $28 \%$ y complejo $58 \%$ (figura 1 ).
Tabla 2. Condición clínica asociada a síndrome confusional.

\begin{tabular}{|c|c|c|c|c|c|}
\hline \multirow[t]{2}{*}{ Condición } & \multicolumn{2}{|c|}{$\begin{array}{c}\text { Confusión } \\
\text { (60 pacientes) }\end{array}$} & \multicolumn{2}{|c|}{$\begin{array}{c}\text { No confusión } \\
\text { (112 pacientes) }\end{array}$} & \multirow[t]{2}{*}{ Valor $\mathrm{p}$} \\
\hline & $\mathrm{n}$ & $\%$ & $\mathrm{n}$ & $\%$ & \\
\hline \multicolumn{6}{|l|}{ Grado funcional } \\
\hline Independiente & 9 & 15 & 53 & 47,3 & \multirow[t]{3}{*}{0,000} \\
\hline Dependiente parcial & 28 & 46 & 41 & 36,6 & \\
\hline Dependiente total & 23 & 39 & 18 & 16,1 & \\
\hline \multicolumn{6}{|l|}{ Nivel cognitivo } \\
\hline Normal & 6 & 10 & 71 & 63,5 & \multirow[t]{4}{*}{0,000} \\
\hline Deterioro leve & 14 & 23 & 28 & 25,0 & \\
\hline Deterioro moderado & 16 & 27 & 4 & 3,5 & \\
\hline Deterioro severo & 24 & 40 & 9 & 8,0 & \\
\hline \multicolumn{6}{|c|}{ Deshidratación moderada/severa } \\
\hline Sí & 25 & 41,7 & 20 & 18 & \multirow[t]{2}{*}{0,001} \\
\hline No & 35 & 58,3 & 92 & 82 & \\
\hline \multicolumn{6}{|l|}{ Diagnóstico de sepsis } \\
\hline Sí & 12 & 20 & 6 & 5,4 & \multirow[t]{2}{*}{0,003} \\
\hline No & 48 & 80 & 106 & 94,6 & \\
\hline
\end{tabular}

Los diagnósticos más frecuentes fueron infección urinaria 30\%, hipertensión arterial $23 \%$, sepsis $17 \%$ y demencia $17 \%$. Los grupos de patologías según presencia de síndrome confusional son presentados en la figura 2 .

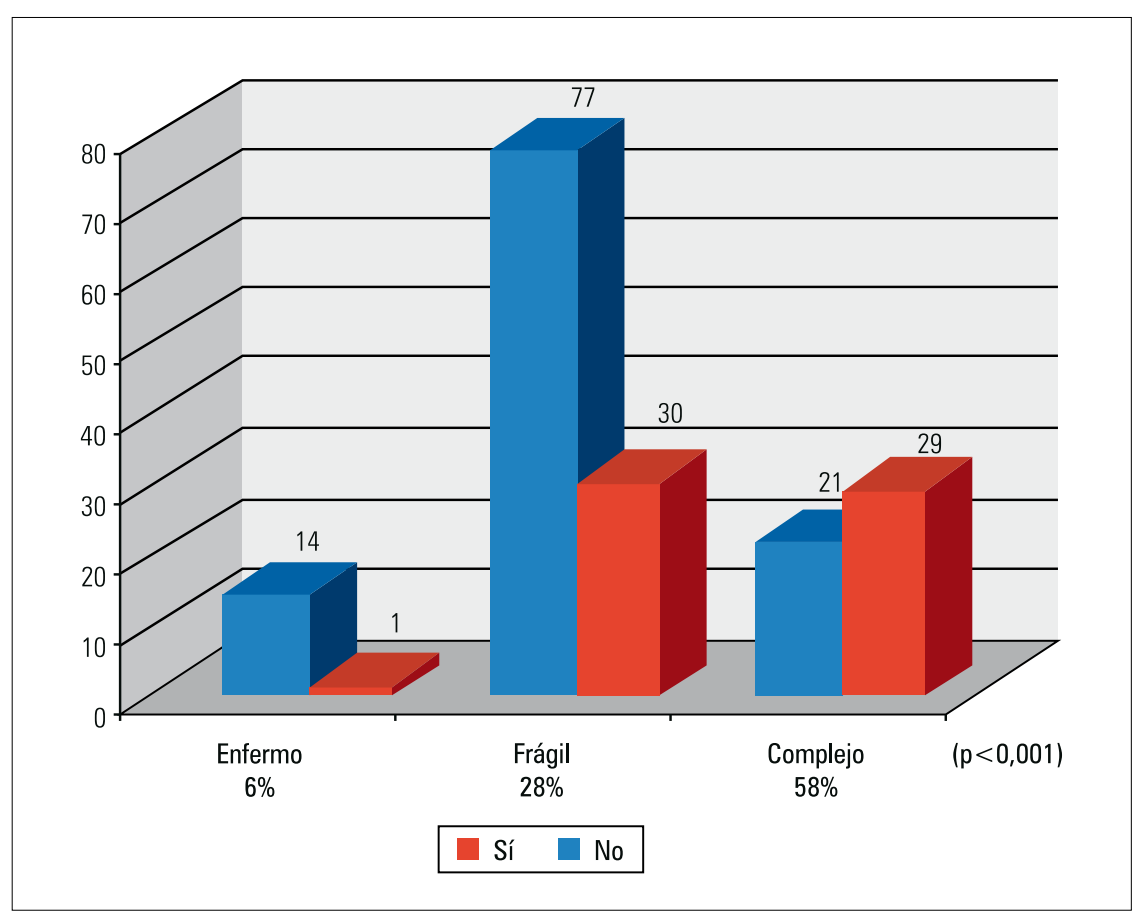

Figura 1. Frecuencia de síndrome confusional agudo según categoría.
No se encontró diferencia en relación a sexo, edad, grupo etario mayor de 80 años, grado de instrucción, estado civil, pluripatología, hospitalización el año previo, polifarmacia, estado nutricional, presencia de sonda urinaria, sonda nasogástrica, destino al egreso de emergencia ni estancia hospitalaria en emergencia.

\section{DISCUSIÓN}

El síndrome confusional agudo no es una entidad clínica aislada, sino la manifestación de diversas patologías, muchas veces multifactorial, pero también de causas previsibles o controlables. Puede ocurrir a cualquier edad, pero es mucho más frecuente en la población adulta mayor $y$, a la vez, subdiagnosticada ${ }^{(5,11,12)}$.

La frecuencia de SCA encontrado en el presente estudio (34,9\%) es similar a lo hallado en hospitales de América Latina ${ }^{(5,11)}$, Norteamérica ${ }^{(8)}$, Europa ${ }^{(10,12,13)}$ y Asia ${ }^{(10)}$, pero con una amplia variabilidad que puede ser explicada al método diagnóstico empleado, al tipo de paciente estudiado o al infradiagnóstico ${ }^{(13)}$. 


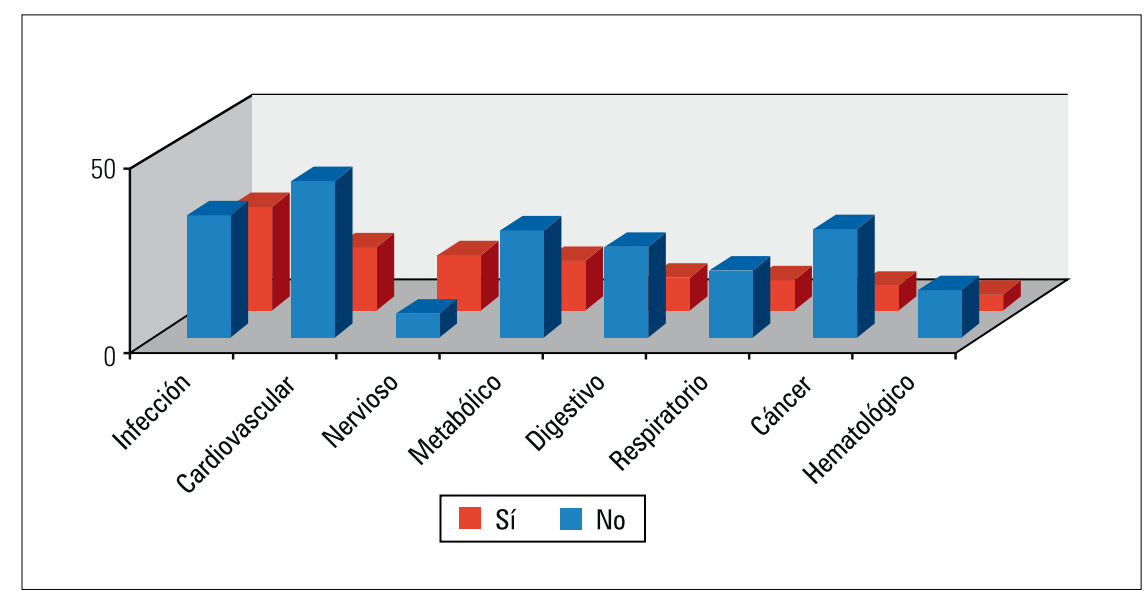

Figura 2. Grupo de patología según presencia de síndrome confusional.

La mayor parte de bibliografía revisada considera síndrome confusional agudo y delirio como sinónimos. Proponemos que el delirio sea considerado como un grado extremo de confusión mental, pues involucra agitación psicomotriz y alucinaciones ${ }^{(1,4)}$.

Existen varios instrumentos para diagnosticar síndrome confusional agudo, pero el CAM es práctico y confiable, según investigaciones realizadas en Europa ${ }^{(12)}$ y Norteamérica ${ }^{(4)}$, con elevada sensibilidad y especificidad.

En el presente estudio no se encontró asociación significativa de edad con síndrome confusional agudo, en contraste con comunicaciones previas de nuestro país ${ }^{(6)}$, Cuba ${ }^{(15)}$, Canadá ${ }^{(7)}$, y España ${ }^{(13)}$, donde hallaron relación directa.

En lo referente al grado de instrucción, destaca la frecuencia baja de analfabetos, debido quizás a que la zona de influencia del hospital de estudio era eminentemente urbana. Tampoco se encontró asociación significativa, en contraste con lo encontrado en hospitales del Ministerio de Salud de nuestro país ${ }^{(6,7)}$.

Para varios autores, la presencia de más de tres enfermedades crónicas por paciente (pluripatología) tuvo influencia significativa para que el adulto mayor desarrolle síndrome confusional ${ }^{(6-8)}$, opuesto a lo encontrado en el presente estudio, debido a que esta categoriza- ción resulta poco adecuada para clasificar al adulto mayor en la actualidad; se ha desarrollado otros índices para medir la comorbilidad en estos pacientes, como el Índice de Charlson.

Similar a lo descrito en la bibliografía, el síndrome confusional agudo es un problema asociado principalmente a complicaciones infecciosas y metabólicas ${ }^{(5-7,11)}$, demostrado por la asociación significativa encontrada con sepsis y deshidratación en el presente trabajo ${ }^{(6,8,13,15)}$.

Episodios previos de síndrome confusional tienen relación significativa con la repetición de este síndrome, reforzando la premisa de que si un adulto mayor presenta un episodio de confusión, lo volverá a tener a corto plazo ${ }^{(5,6,13)}$.

Se confirma que la demencia es un factor muy frecuente e importante para desarrollar síndrome confusional agudo en el adulto mayor ${ }^{(6,13)}$. Incluso, se comunica que la presentación de nuevos episodios de confusión aguda empeora el pronóstico y acelera el deterioro mental de estos pacientes con demencia ${ }^{(16)}$.

A diferencia de una comunicación nacional ${ }^{(6)}$, el estado nutricional al ingreso no tuvo relación significativa con el síndrome confusional, debido probablemente a que la población evaluada tenía mayor acceso al sistema de salud y proporción baja de malnutrición.
El grado de dependencia funcional y deterioro del nivel cognitivo presentaron asociación significativa con síndrome confusional agudo, relacionado también a mayor morbilidad y deterioro del paciente. Similar ha sido lo informado en hospitales locales ${ }^{(5,7)}$.

Las categorías de adulto mayor adoptadas por el Ministerio de Salud de nuestro país (saludable, enfermo, frágil y complejo) presentan diferencia significativa en la presentación del síndrome confusional agudo; esto se explica porque en esta categorización se incluye factores como estado mental, funcional, y otros que tienen relación directa con el desarrollo de este síndrome ${ }^{(14)}$.

Similar a lo hallado en Canadá (2002) ${ }^{(8)}$, el destino y la estancia hospitalaria de los pacientes estudiados no está influenciada por la presencia de síndrome confusional agudo. Sin embargo, difiere de lo encontrado en un hospital local (5), donde hubo mayor estancia hospitalaria en pacientes con síndrome confusional, explicado quizás porque el presente estudio solo midió el egreso de emergencia y no el alta definitiva del hospital.

Como principales limitaciones encontramos un importante subregistro en la historia clínica hospitalaria (antigua y de ingreso), escaso interés del personal de salud en el tema y el sesgo por olvido de antecedentes por parte del paciente o cuidador.

En conclusión, se halló frecuencia alta $(34,9 \%)$ del síndrome confusional agudo en pacientes adultos mayores no críticos que se hospitalizan en el servicio de emergencia del Hospital Nacional Edgardo Rebagliati Martins Lima-Perú, 2010. Los principales factores asociados a síndrome confusional agudo en el adulto mayor fueron antecedente de demencia, deterioro cognitivo previo, síndrome confusional previo, dependencia funcional, categoría geriátrico complejo, deshidratación moderada-severa y diagnóstico de sepsis al ingreso. 


\section{REFERENCIAS BIBLIOGRÁFICAS}

1. Inouye SK. Delirium in older persons. N Engl J Med. 2006;354:1157-65. [citado 2010 may 12]. Disponible en: http://www.nejm.org.

2. INEI. Perfil Sociodemográfico del Perú. Censos Nacionales 2007: XI de población y VI de vivienda, 2da ed. Lima 2008. [Citado: enero 2012]. Disponible en: www.inei.gob.pe/Anexos/libro.pdf.

3. Varela L, Chávez H, Gálvez M, Méndez F. Funcionalidad en el adulto mayor previa a su hospitalización a nivel nacional. Rev Med Hered. 2005;16:165-71.

4. Wei LA, Fearing MA, Sternberg EJ, Inouye SK. The Confusion Method (CAM): a systematic review of current usage. J Am Geriatr Soc. 2008;56(5):823-30. [Citado 2011 jul 9]. Disponible en: http://www.ncbi. nlm.nih.gov/pmc/articles/PMC2585541/.

5. Ortiz P, Chávez JC, Chávez H, Varela LF. Delirio en el paciente adulto mayor hospitalizado: presentación clinica y morbimortalidad. Rev Soc Peru Med Inter. 2002;14(4):184-90.

6. Valera L, Chávez H, Gálvez M, Méndez F. Características del deterioro cognitivo en el adulto mayor hospitalizado a nivel nacional. Rev Soc Peru Med Inter. 2004;17(2):37-42.

7. Lama J, Valera L, Ortiz PJ. Prevalencia y factores de riesgo del estado confusional agudo en el adulto mayor en una sala de emergencias médicas. Rev Med Hered. 2002;13(1):10-8.
8. Dubois MJ, Bergeron N, Dumont M, Dial S, Skrobik $Y$. Delirium in an intensive care unit: a study of risk factors. Intensive Care Med. 2001;27(8):1297-304.

9. Grover S, Subodh BN, Avasthi A, Chakrabarti S, Kumar S, Sharan P, et al. Prevalence and clinical profile of delirium: a study from a tertiary-care hospital in north India. General Hospital Psychiatry. 2009;31:25-9.

10. Van Rompaey B, Elseviers MM, Schuurmans MJ, Shortridge-Baggett LM, Truijen S, Bossaert L. Risk factors for delirium in intensive care patients: a prospective cohort study. Critical Care. 2009;13(3):7789.

11. Carrasco M, Hoyl T, Marín PP, Hidalgo J, Lagos $C$, Longton $C$, et al. Subdiagnóstico de delirum en adultos mayores hospitalizados. Rev Méd Chile. 2005;133:1449-54. [Citado: julio 2009]. Disponible en: http://www.scielo.cl/scielo.php?pid=S003498872005001200006\&script=sci_arttext

12. Laurila JV, Pitkala KH, Strandberg TE, Tilvis RS. Detection and documentation of dementia and delirium in acute geriatric wards. General Hospital Psychiatry. 2004;26:31-5.

13. Formiga F, Marcos E, Sole A, Valencia E, LoraTamayo J, Pujol R. Sindrome confusional agudo en pacientes ancianos ingresados por patología médica. Rev Clin Esp. 2005;205(10):484-8. [Citado: julio 2009]. Disponible en: http://www.sciencedirect. com/science/article/pii/S0014256505726187
14. Ministerio de Salud. Guía técnica para el llenado de la historia clínica de atención integral de salud del adulto mayor. Perú 2006. 26 pp. [Citado: diciembre 2009]. Disponible en: http://www.minsa.gob.pe/ normaslegales/2006/RM1147-2006.pdf

15. Bayarre HD. Prevalencia y factores de riesgo de discapacidad en ancianos. Ciudad de La Habana y Las Tunas. 2000. [Tesis Doctoral]. Escuela Nacional de Salud Pública de Cuba. 2003:125 pp.

16. Fong TG, Jones RN, Shi P, Marcantonio ER, Yap L, Rudolph JL, et al. Delirium accelerates cognitive decline in Alzheimer disease. Neurology. 2009;72:1570-5.

Artículo recibido el 6 de abril 2012 y aceptado para publicación el 20 de abril de 2013.

Fuente de financiamiento: Autofinanciado.

Conflicto de intereses: No presenta conflicto de intereses.

Correspondencia:

Dr. José Percy Amado Tineo.

Jr. Belisario Flores 238 Dpto 301 - Lince. Lima 14.

Correo electrónico: jpamadot@hotmail.com

Teléfono: 990452547. 